\title{
Novel microwell-based spectrophotometric assay for determination of atorvastatin calcium in its pharmaceutical formulations
}

\author{
Tanveer A Wani ${ }^{1}$, Nasr Y Khalil', Hamdy M Abdel-Rahman² and Ibrahim A Darwish ${ }^{1 *}$
}

\begin{abstract}
The formation of a colored charge-transfer (CT) complex between atorvastatin calcium (ATR-Ca) as a n-electron donor and 2, 3-dichloro-5,6-dicyano-1,4-benzoquinone (DDQ) as a r-electron acceptor was investigated, for the first time. The spectral characteristics of the $\mathrm{CT}$ complex have been described, and the reaction mechanism has been proved by computational molecular modeling. The reaction was employed in the development of a novel microwell-based spectrophotometric assay for determination of ATR-Ca in its pharmaceutical formulations. The proposed assay was carried out in 96-microwell plates. The absorbance of the colored-CT complex was measured at $460 \mathrm{~nm}$ by microwellplate absorbance reader. The optimum conditions of the reaction and the analytical procedures of the assay were established. Under the optimum conditions, linear relationship with good correlation coefficient (0.9995) was found between the absorbance and the concentration of ATR-Ca in the range of 10-150 $\mu \mathrm{g} /$ well. The limits of detection and quantitation were 5.3 and $15.8 \mathrm{\mu g} /$ well, respectively. No interference was observed from the additives that are present in the pharmaceutical formulation or from the drugs that are co-formulated with ATR-Ca in its combined formulations. The assay was successfully applied to the analysis of ATR-Ca in its pharmaceutical dosage forms with good accuracy and precision. The assay described herein has great practical value in the routine analysis of ATR-Ca in quality control laboratories, as it has high throughput property, consumes minimum volume of organic solvent thus it offers the reduction in the exposures of the analysts to the toxic effects of organic solvents, and reduction in the analysis cost by 50-fold. Although the proposed assay was validated for ATR-Ca, however, the same methodology could be used for any electron-donating analyte for which a CT reaction can be performed.
\end{abstract}

\section{Background}

Atorvastatin calcium (ATR-Ca); [(R-( $\left.\left.\mathrm{R}^{*}, \mathrm{R}^{*}\right)\right]$-2-(4-fluorophenyl) $-\beta, \delta$, dihydroxy-5-(1-methylethyl)-3-phenyl-4[(phenyl-amino)-carbonyl]-1H-pyrrole-1-heptanoic acid calcium salt (Figure 1), is a second generation synthetic 3hydroxy-3-methylglutaryl-coenzyme A (HMG-CoA) reductase inhibitor [1]. It exerts its action by specifically inhibiting the HMG-CoA reductase, the enzyme that catalyzes the conversion of HMG-CoA to mevolanate, which is the early rate-limiting step in the biosynthesis of cholesterol in the body. Inhibition of the enzyme decreases de novo cholesterol synthesis, increasing expression of lowdensity lipoprotein receptors (LDL receptors) on hepatocytes. This increases the uptake of LDL by the hepatocytes,

\footnotetext{
* Correspondence: idarwish@ksu.edu.sa

'Department of Pharmaceutical Chemistry, College of Pharmacy, King Saud University, P.O. Box 2457, Riyadh 11451, Saudi Arabia

Full list of author information is available at the end of the article
}

decreasing the amount of LDL-cholesterol in the blood. ATR-Ca also reduces blood levels of triglycerides and slightly increases levels of HDL-cholesterol. ATR-Ca is the most efficient and frequently prescribed drug for the treatment of hypercholesterolaemia [2].

Atorvastatin calcium is official in USP 34 [3] and it is analyzed in its bulk by HPLC, however the USP does not describe an assay method for ATR-Ca in its dosage forms. This beside the therapeutic importance of ATR-Ca was behind the growing interest in the development of analytical methods for its determination in its pharmaceutical formulations. In general, spectrophotometry is the most widely used technique in pharmaceutical analysis because of its inherent simplicity and wide availability in most quality control laboratories [4-9]. However, the spectrophotometric methods that have been reported for determination of ATR-Ca in its pharmaceutical formulations [10-16] suffer from major drawbacks. These drawbacks 


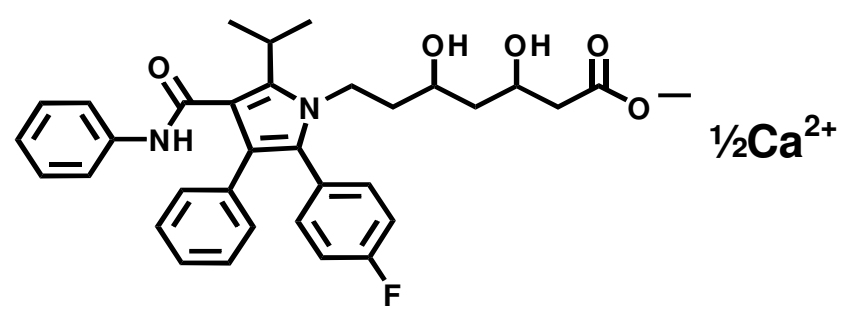

Atorvastatin calcium (ATR-Ca)<smiles>O=C1[C@H](CCC(O)c2ccc(F)cc2)[C@H](c2ccc(O)cc2)N1c1ccc(F)cc1</smiles>

Ezetimibe<smiles>CC(=O)Oc1ccccc1C(=O)O</smiles>

Aspirin<smiles>CC(C)OC(=O)C(C)(C)Oc1ccc(C(=O)c2ccc(Cl)cc2)cc1</smiles>

Fenofibrate<smiles>CCOC(=O)C1=C(COCCN)NC(C)=C(C(=O)OC)C1c1ccccc1Cl</smiles>

Amlodipine besylate

Figure 1 The chemical structure of atorvastatin calcium (ATR-Ca) and the co-formulated drugs.

include decreased selectivity due to measuring the native light absorption of ATR-Ca in the blue-shifted ultraviolet region, which might be subjected to interferences [12], employment of multiple-steps of non-selective oxidation reactions [13-15], and tedious liquid-liquid extraction procedures using large volumes of organic solvents in the methods based on formation of ion-pair associates [16]. Therefore, the development of a new alternative spectrophotometric method for determination of ATR-Ca in its pharmaceutical formulations is very essential.

The molecular interactions between the electron-donating pharmaceutical compounds and electron-accepting reagents are generally associated with the formation of intensely colored CT complexes, which usually absorb radiations in the visible region. The rapid formation of these complexes leads to their widespread utility in the development of visible spectrophotometric methods for analysis of many pharmaceutical compounds [17-23]. Literature survey revealed that the CT reaction of ATR-Ca has not been investigated yet. As well, in a previous study, Darwish IA [21] demonstrated that the alkali salts of carboxylic pharmaceutical compounds have excellent electron-donating ability, thus it is anticipated that ATR-Ca (the active ingredient of its dosage forms) will exhibit electron-donating capabilities. These facts promoted our interest in employment of the CT-reaction as a basis for the development of a new spectrophotometric method for determination of ATR-Ca. However, all the conventional CT-based spectrophotometric methods that have been reported so far are not automated and consequently their 
throughput is low, thus their applications in pharmaceutical quality control laboratories are limited. Moreover, these methods suffer from the consumption of large volumes of organic solvents, which leads to high analysis cost, and more importantly, the incidence of exposure of the analysts to the toxic effects of the organic solvents [24-28].

For these reasons, the present research proposal is devoted to investigate the CT reaction of ATR-Ca, and its employment in the development of novel non-conventional spectrophotometric assay with high analysis throughput and can reduce the consumption of organic solvents in the determination of ATR-Ca in its pharmaceutical formulations.

\section{Experimental Apparatus}

Microwell-plate absorbance reader (ELx 808, Bio-Tek Instruments Inc. Winooski, USA) was used for all the measurements in 96-microwell plates. UV-1601 PC (Shimadzu, Kyoto, Japan) ultraviolet-visible spectrophotometer with matched $1 \mathrm{~cm}$ quartz cells was used for recording the absorption spectra. 96-Microwell plates were a product of Corning/Costar Inc. (Cambridge, USA). Finnpipette adjustable 8-channel-pipette was obtained from Sigma Chemical Co. (St. Louis, MO, USA).

\section{Chemicals and dosage forms}

ATR-Ca and amlodipine besylate were obtained from Pfizer Inc. (New York, USA). Ezetimibe was obtained from AK Scientific Inc. (California, USA). Fenofibrate, and aspirin were obtained from Sigma Chemical Co. (St. Louis, USA). DDQ (Merck, Germany) was $0.1 \%(w / v)$ in methanol and it was prepared fresh daily. Lipitor tablets (Parke Davis, Germany) and Lipicure-10 tablets (INTAS Pharmaceuticals, India) labeled to contain $10 \mathrm{mg}$ ATR-Ca were obtained from the local market.

\section{Preparation of atorvastatin from ATR-Ca}

An accurately weighed amount ( $25 \mathrm{mg}$ ) of ATR-Ca was dissolved in $10 \mathrm{ml}$ water in a test tube. The solution was transferred into a 100-ml separating funnel, then rendered acidic with $10 \% \mathrm{HCl}$. The liberated atorvastatin (acid form) was extracted by chloroform. The chloroformic solution was evaporated under nitrogen, and the obtained residue was reconstituted in methanol. The methanolic solution was tested for its ability to exhibit CT reaction.

\section{Preparation of standard and tablet solutions Preparation of stock standard solutions}

Into a 5-ml calibrated flask, $10 \mathrm{mg}$ of ATR-Ca was accurately weighed, dissolved in $2 \mathrm{ml}$ methanol, and completed to volume with the same solvent. This stock solution was diluted with methanol to obtain the suitable concentrations that lie in the linear range of the assay.

\section{Preparation of tablet sample solutions}

Twenty tablets were weighed and finely powdered. A quantity of the powder equivalent to $20 \mathrm{mg}$ of ATR-Ca was transferred into a 10-ml calibrated flask, dissolved in $4 \mathrm{ml}$ methanol, swirled and sonicated for $5 \mathrm{~min}$, completed to volume with the methanol, shaken well for $15 \mathrm{~min}$, and filtered. The first portion of the filtrate was rejected, and a measured volume of the filtrate was diluted quantitatively with methanol to yield the suitable concentrations that lie in the linear range of the assay.

\section{General analytical procedure}

Accurately measured aliquots $(100 \mu \mathrm{l})$ of the standard or sample solution containing varying amounts of ATR-Ca (10-150 $\mu \mathrm{g})$ were transferred into wells of 96-microwell assay plates. One hundred microliters of DDQ solution $(0.1 \%, \mathrm{w} / \mathrm{v})$ was added, and the reaction was allowed to proceed at room temperature $\left(25 \pm 1^{\circ} \mathrm{C}\right)$ for $5 \mathrm{~min}$. The absorbances of the resulting solutions were measured at $460 \mathrm{~nm}$ by the microwell-plate reader. Blank wells were treated similarly except $100 \mu \mathrm{l}$ of methanol was used instead of sample, and the absorbances of the blank wells were subtracted from those of the other wells.

\section{Determination of molar ratio}

The Job's method of continuous variation [29] was employed. Master equimolar solutions $\left(2 \times 10^{-3} \mathrm{M}\right)$ of each of ATR-Ca and DDQ were prepared. Series of $200 \mu \mathrm{l}$ portions of the master solutions of ATR-Ca with DDQ were made up comprising different complementary ratios $(0: 10,1: 9, \ldots \ldots . . ., 9: 1,10: 0$, inclusive) in each well of the 96-microwell assay plate. The reaction was allowed to proceed at room temperature $\left(25 \pm 1^{\circ} \mathrm{C}\right)$ for $5 \mathrm{~min}$. The absorbances of the developed colors were measured at $460 \mathrm{~nm}$ by the microwell-plate reader against blank wells treated similarly except methanol was used instead of ATR-Ca sample. The measured absorbances were plotted as a function of ATR-Ca mole fraction. The generated plot was used for determination the molar ration of ATRCa:DDQ.

\section{Molecular modeling for the CT complex of ATR-Ca with DDQ}

The molecular modeling for the CT complex was performed by using CS Chem3D Ultra, version 9 (Cambridge Soft Corporation, Cambridge, MA, USA) implemented with molecular orbital computations software (MOPAC), and molecular dynamics computations software (MM2).

\section{Results and Discussion}

Design of the proposed assay and strategy for its development

The proposed assay was designed to employ 96-microwell assay plate as the CT reaction was carried out in microwells of the assay plate $(200-\mu$ l reaction volume) 
instead of the conventional volumetric flasks $(10,000-\mu \mathrm{l}$ volume). The solutions were dispensed by 8 -channel pipette, and the absorbances of the colored CT complex were measured by microwell-plate absorbance reader instead of the conventional spectrophotometer.

In the present study, ATR-Ca was selected based on its therapeutic importance, clinical success, and the expected electron-donating ability. This selection was supported by a previous study made by Darwish IA [21], which demonstrated the excellent electron-donating property of alkali salts of carboxylic acid pharmaceutical compounds. Previous studies involving CT reactions with polyhalo-/polycyanoquinone electron $n$-acceptors revealed that DDQ is one of the most efficient reagents in terms of its reactivity $[21,30]$. Furthermore, its CT reaction with electron-donating analytes is instantaneous $[21,22]$. For these reasons, DDQ was used as electron acceptor in the development of the proposed assay. The 96-microwell design of the proposed assay was based on the previous success of Darwish et al [31] in the utility of this design for determination of some other pharmaceuticals.

\section{Reaction and spectral characteristics}

The interaction of ATR-Ca with DDQ was allowed to proceed at room temperature, and the absorption spectrum of the produced chromogen was recorded. ATRCa gave red colored chromogen showing absorption maximum at $460 \mathrm{~nm}$ (Figure 2). This band was attributed to the formation of the radical anion $\mathrm{DDQ}^{-}$[32], which was probably formed by the dissociation of an original donor-acceptor (D-A) complex:

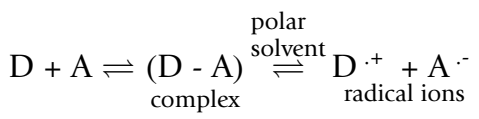

Further support of this assignment was provided by the absorption maxima with those of DDQ radical anion produced by the iodide reduction assay [32]. The dissociation of the (D-A) complex was promoted by the high ionizing power of the polar solvent and the resulting peaks in the absorption spectra of drug-acceptor reaction mixtures were similar to the maxima of the radical anions of the acceptors obtained by the iodide reduction assay [32].

\section{Optimization of experimental conditions}

The optimization of experimental conditions affecting the reaction in the 96-well format was investigated by altering each reaction variable in a turn while keeping the others constant. In all cases, measurements were carried out at $460 \mathrm{~nm}$, as this maximum gave the highest absorptivities and ultimately the highest analytical sensitivities. The results of variations in the DDQ concentrations indicated that $100 \mu \mathrm{l}$ of $0.1 \%(\mathrm{w} / \mathrm{v})$ was the optimum DDQ concentration, as this concentration gave the highest absorbances. Previous studies [33] demonstrated that the interaction of electron-donors with DDQ in polar solvents (e.g. methanol and acetonitrile) produces CT complexes with molar absorptivity values higher than those produced in non-polar solvents (e.g. chloroform). Different polar solvents were tested as reaction solvent; these solvents were methanol, ethanol, 1-propanol, 1-butanol, and acetonitrile. The obtained

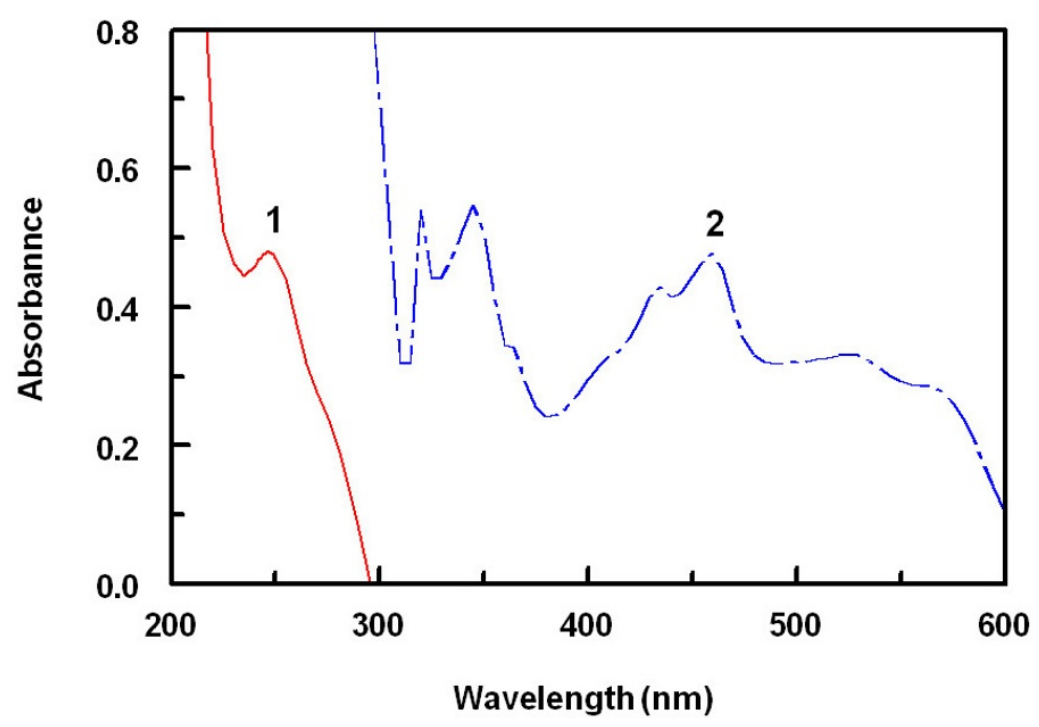

Figure 2 Absorption spectrum of (1) ATR-Ca $(125 \mu \mathrm{g} / \mathrm{ml})$ and (2) reaction product of ATR-Ca (125 $\mu \mathrm{g} / \mathrm{ml})$ with DDQ $(0.1 \%$, w/v) 
absorbances were $0.493,0.480,0.464,0.432$, and 0.483 , respectively. These values indicated that methanol offered the highest sensitivity, therefore it was selected for the subsequent experiments. As well, these solvents has no any harmful effect on the transparency of the microwells. The optimum reaction time was determined by monitoring the color development in the microwells at room temperature $\left(25 \pm 1^{\circ} \mathrm{C}\right)$. Complete color development was attained instantaneously, however for higher precision readings, the reaction was allowed to proceed for $5 \mathrm{~min}$. The developed color remained stable at room temperature for at least a further $30 \mathrm{~min}$.

\section{Molar ratio of the reaction, molecular modeling, and proposing the site of interaction}

Job's method of continuous variation was used for determining the molar ratio of ATR-Ca to DDQ. The obtained Job's plot was given in Figure 3, from which it was concluded that the ATR-Ca:DDQ ratio is 1:2. This indicated that two moles of DDQ interacted with one mole of ATR$\mathrm{Ca}$. Considering the divalent calcium ion, the reaction was postulated to proceed as 1:1 ratio for DDQ with atorvastatin anion via only one site of interaction in spite of the presence of more than one possible electron-donating sites; $\mathrm{OH}$ of the side chain and the nitrogen atom of pyrole ring. The site of interaction was preliminarily postulated to be the anionic carboxylate moiety of ATR-Ca. To prove this suggestion, the $\mathrm{CT}$ reaction was carried out using the parent atorvastatin acid. It was found that atorvastatin did not exhibit $\mathrm{CT}$ reaction indicating that the anionic carboxylate moiety of ATR-Ca was the site of CT interaction with DDQ. For further support of this assumption, modeling for the CT complex was performed. Atorvastatin anion and DDQ were energy-minimized alone and both together. It was found that the highest electron densities in the atorvastatin molecule are located on the two oxygen atoms of the carboxylate anion followed by the two

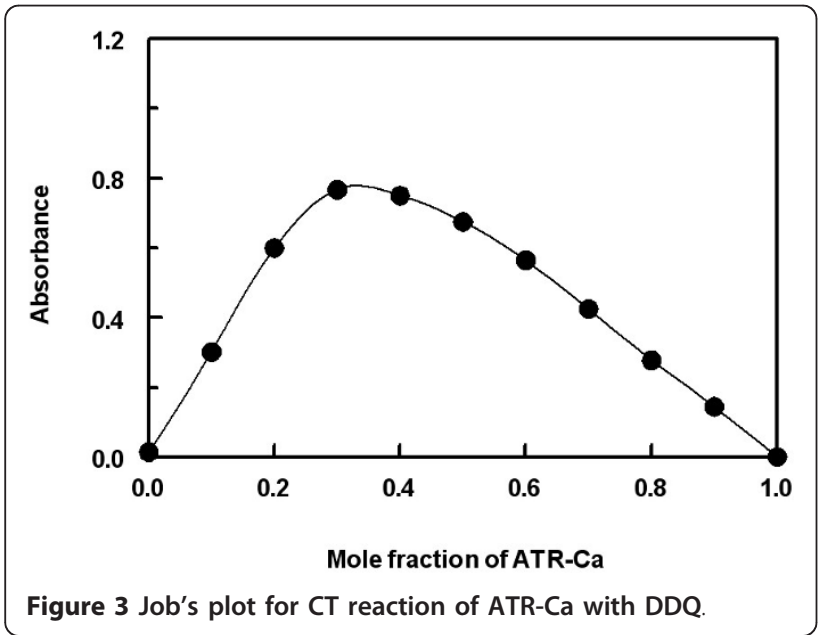

alcoholic oxygen atoms of the side chain. The total charges on each of the two oxygen atoms of the carboxylate anion were found to be -0.6234 and -0.5975 . Therefore, it is expected that the carboxylate anion moiety of ATR-Ca will move toward DDQ to form the CT complex (Figure 4). These facts, taking the molar ratio in account, confirmed that only the carboxylate oxygens are involved in the complex formation, and the reaction proceeded as described in Figure 5. The nitrogen atom of the pyrole ring and $\mathrm{OHs}$ of the side chain did not contribute in the $\mathrm{CT}$ reaction based on the fact that certain electron density was required for achievement of a successful electron transfer [34].

\section{Validation of the proposed assay Linearity and sensitivity}

Under the above mentioned optimum reaction conditions, the calibration curve for the analysis of ATR-Ca by the proposed assay was constructed by plotting the absorbances as a function of the corresponding concentrations. The regression equation for the results was derived using the least-squares method. Beer's law plot $(n=5)$ was linear with very small intercept $(0.0045)$ and good correlation coefficient (0.9995) in the concentration range of 10 - 150 $\mu \mathrm{g} / \mathrm{well}(100 \mu \mathrm{l})$. The limits of detection (LOD) and quantitation (LOQ) were determined [35] using the formula: $\mathrm{LOD}$ or LOQ $=\kappa \mathrm{SDa} / \mathrm{b}$, where $\kappa=3$ for LOD and 10 for LOQ, SDa is the standard deviation of the intercept, and b is the slope. The LOD and LOQ values were 5.3 and $15.8 \mu \mathrm{g} /$ well $(100 \mu \mathrm{l})$, respectively. The quantitative parameters of the proposed assay are given in Table 1 .

\section{Accuracy and precision}

Accuracy of the proposed assay was assessed by analytical recovery studies. Recovery was determined by the standard addition method. Known amounts of ATR-Ca were added to pre-determined drug-containing pharmaceutical formulation, and then determined by the proposed assay. The mean analytical recovery was calculated and found to be $97.03-99.91 \pm 0.33-3.49 \%$ indicating the accuracy of the proposed assay.

The precisions of the proposed assay were determined on samples of drug solutions at three concentration levels for each drug (Table 2) by analyzing 5 replicates of each sample as a batch in a single assay run. The relative standard deviations (RSD) did not exceed $1.48 \%$ (Table 2) proving the high precision of the assay for the routine application in quality control laboratories. This high level of precision was attributed to the accuracy of the volumes that have been concomitantly dispensed in the microwells by multi-channel pipettes, and completeness of the reaction the small volume $(200 \mu \mathrm{l})$.

\section{Selectivity}

The proposed assay has the advantages that the measurements are performed in the visible region, away from the 


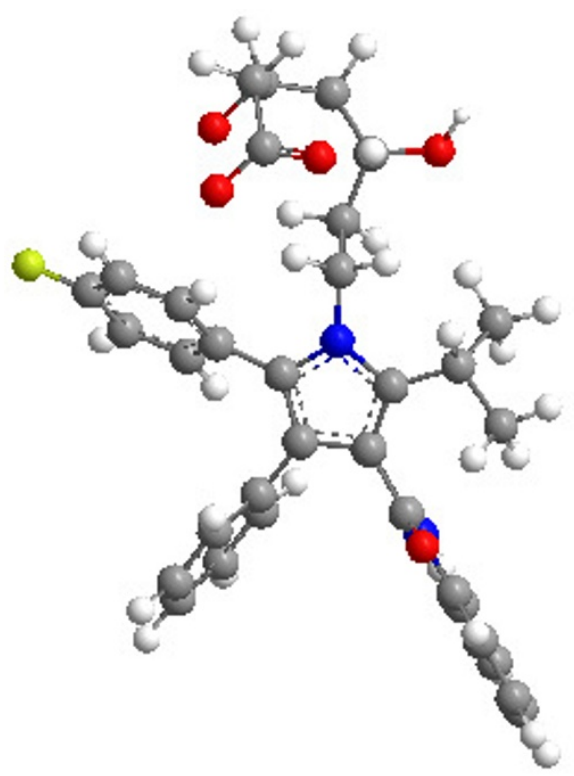

Atorvastain anion

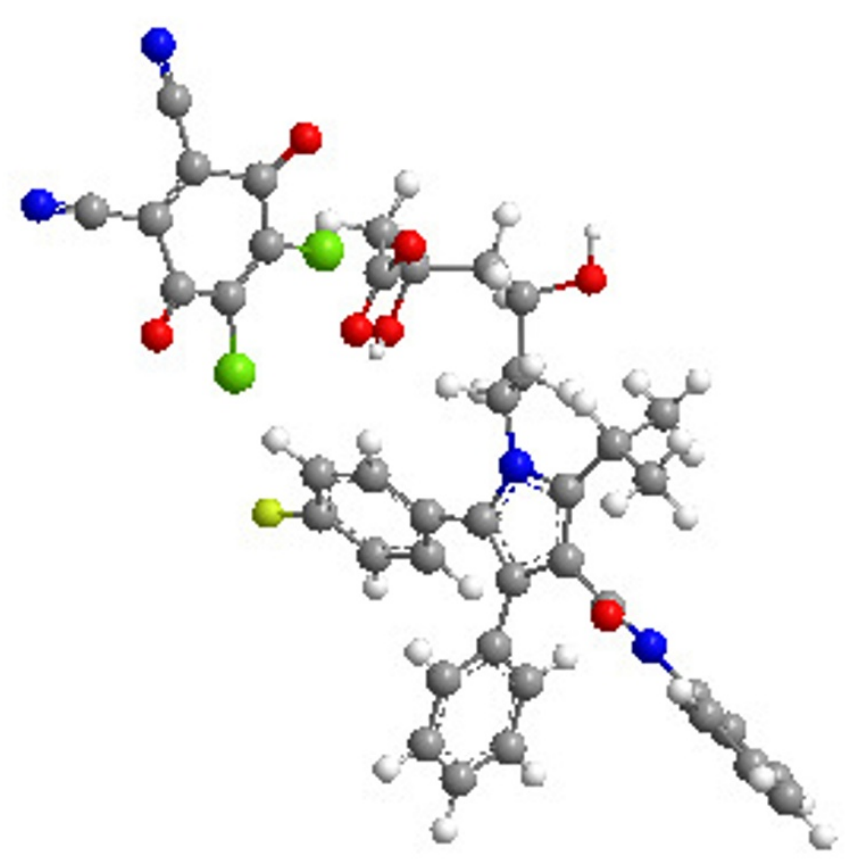

CT complex of atorvastatin anion with DDQ

Figure 4 Energy-minimized atorvastatin anion and its CT complex with DDQ

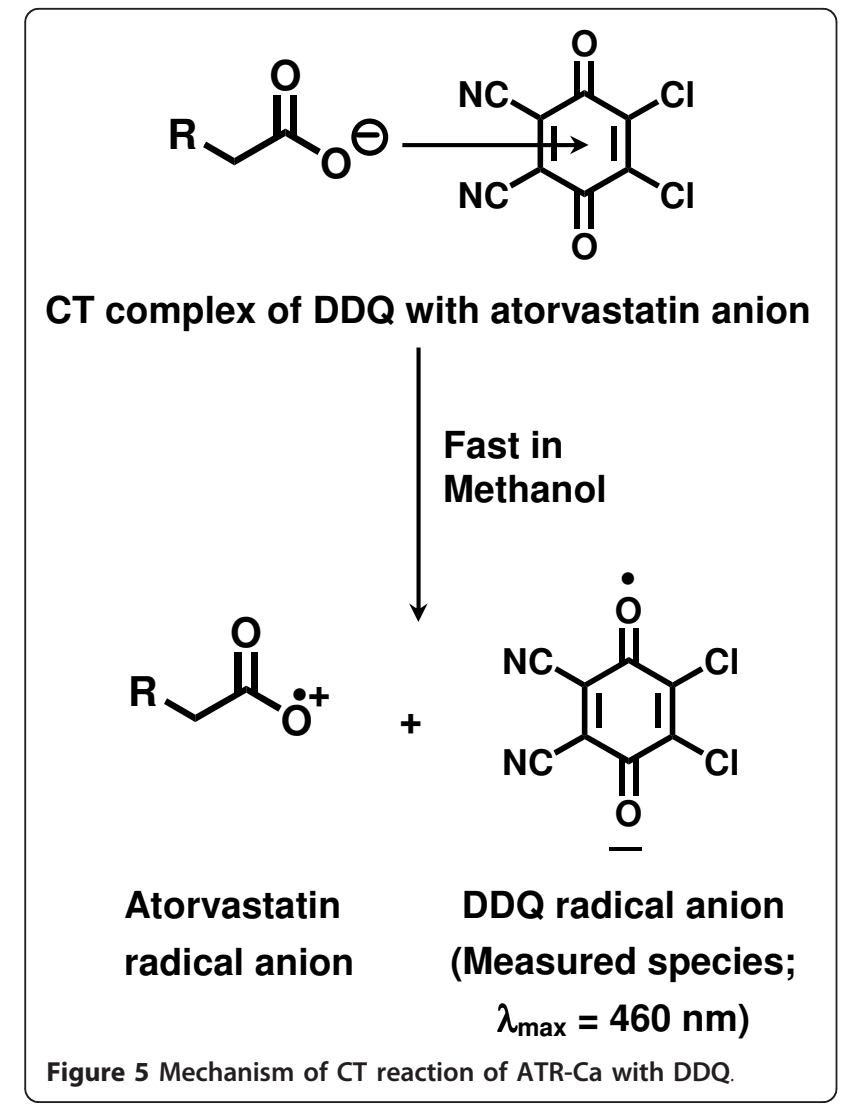

UV-absorbing interfering substances that might be coextracted from dosage forms that contain ATR-Ca. The interference from the congenital drugs that is co-formulated with ATR-Ca in some of its dosage forms was studied. These drugs were aspirin [36], fenofibrate [37], ezetimibe [38] and amlodipine besylate [39,40]; the chemical structures of these drugs are given in Figure 5. Potential interferences of these drugs were studied in a ratio which is normally present in their combined dosage forms. No interferences from these drugs were found with ATR-Ca in the proposed assay. Although, amlodipine has basic character, however it is co-formulated with ATR-Ca as besylate salt that did not show any ability for CT reaction with DDQ under the proposed assay

Table 1 Quantitative parameters for the analysis of ATRCa by the proposed assay

\begin{tabular}{|c|c|}
\hline Parameter & Value \\
\hline Range $(\mu \mathrm{g} / \text { well })^{a}$ & $10-150$ \\
\hline Intercept & 0.0045 \\
\hline Slope & 0.0066 \\
\hline Correlation coefficient & 0.9995 \\
\hline Molar absorptivity $(\mathrm{L} / \mathrm{mol} / \mathrm{cm})$ & 675 \\
\hline $\operatorname{LOD}(\mu \mathrm{g} / \text { well })^{a}$ & 5.3 \\
\hline LOQ ( $\mu \mathrm{g} /$ well) ${ }^{a}$ & 15.8 \\
\hline
\end{tabular}

\footnotetext{
${ }^{a}$ Each well contained $100 \mu$ l of ATR-Ca sample.
} 
Table 2 Precision of the proposed assay at different ATR-Ca concentrations

\begin{tabular}{lll}
\hline Concentration $(\boldsymbol{\mu g} /$ well) & Relative standard deviation \\
\cline { 2 - 3 } & Within-assay, $\mathbf{n}=\mathbf{5}$ & Between-assays, $\mathbf{n}=\mathbf{5}$ \\
\hline 25 & 1.49 & 1.84 \\
50 & 1.36 & 1.60 \\
125 & 1.11 & 1.17 \\
\hline
\end{tabular}

conditions. As well, no interference was observed from the excipients with the proposed assay as indicated from the obtained good recovery (mentioned above). The absence of interference from the excipients, even though they contain basic component(s) was attributed to the extraction of the ATR-Ca tablets prior to the analysis with methanol in which the excipients do not dissolve.

\section{Application of the proposed assay in the analysis of pharmaceutical formulations}

The commercially available pharmaceutical formulations of ATR-Ca were subjected to the analysis by the proposed and reported methods [15] and the obtained results were then statistically compared with each other. The mean percentage recoveries, relative to the labeled amounts, obtained by the proposed assay were 97.24 \pm 1.50 and $99.82 \pm 1.05 \%$ for lipitor and lipicure-10 tablets, respectively (Table 3 ). In the t- and F-tests, no significant differences were found between the calculated and theoretical values of both the proposed and the reported assays at 95\% confidence level. This indicated similar accuracy and precision in the analysis by the proposed and reported methods.

\section{Conclusions}

The present study described the development and validation of a novel microwell-based spectrophotometric assay for the determination of ATR-Ca based on its CT reaction with DDQ reagent. In this assay, the $\mathrm{CT}$ reaction was carried out in 96-microwell plates $(200-\mu \mathrm{l}$ reaction volume) instead of the conventional volumetric flasks (10,000- $\mu$ l volume). The absorbances were measured by microwell-plate reader instead of the conventional spectrophotometer. The assay described herein offered the following advantages:

- Reduction in the consumption of organic solvents in the CT-based spectrophotometric analysis, accordingly reduction in the exposures of the analysts to the toxic effects of organic solvents.
- Reduction in the analysis cost by 50-folds which can be reflected on the price for the finished dosage forms, thus it can reduce the expenses for the medications.

- Providing a high throughput analytical methodology that can facilitate the processing of large number of samples in a relatively short time. This property was attributed to the use of multi-channel pipettes for efficient dispensing of the solutions, carrying out the analytical reaction in 96-well plates (as reaction vessels), and measuring the color signals in the 96 wells at $\sim 30 \mathrm{sec}-$ onds by the plate reader.

- The advantages of the proposed assay and in addition to automation could be reached by application of Flow Injection Analysis.

- Although the proposed assay was developed and validated for ATR-Ca, however, it is also anticipated that the same methodology could be used for essentially any analyte that can exhibit $\mathrm{CT}$ reaction.

\section{Abbreviations}

CT: charge-transfer; ATR-Ca: atorvastatin calcium; DDQ: 2,3-dichloro-5,6dicyano-1,4-benzoquinone; HMG-CoA: 3-hydroxy-3-methylglutaryl-coenzyme A; LDL: low-density lipoprotein; LOD: limit of detection; LOQ: limit of quantification; SD: standard deviation; RSD: relative standard deviation.

\section{Acknowledgements}

The authors extend their appreciation to the Deanship of Scientific Research at King Saud University for funding the work through the research group No. RGP-VPP-065.

\section{Author details}

'Department of Pharmaceutical Chemistry, College of Pharmacy, King Saud University, P.O. Box 2457, Riyadh 11451, Saudi Arabia. ${ }^{2}$ Department of Medicinal Chemistry, Faculty of Pharmacy, Assiut University, Assiut 71526, Egypt.

\section{Authors' contributions}

TW participated in the design of the study and conducting the optimization of the assay conditions and validation. NK participated in the assay design and conducted the assay validation and analysis of dosage forms. HA carried out the molecular modeling of the ATR-Ca:DDQ charge-transfer complex. ID

Table 3 Analysis of ATR-Ca in its pharmaceutical formulations by the reported and proposed methods

\begin{tabular}{|c|c|c|c|c|}
\hline \multirow[t]{2}{*}{ Pharmaceutical formulation } & \multicolumn{2}{|l|}{ Content $\left(\% \pm\right.$ SD) ${ }^{a}$} & \multirow[t]{2}{*}{ t-Value $^{b}$} & \multirow[t]{2}{*}{ F-value $^{b}$} \\
\hline & Proposed method & Reported method & & \\
\hline Lipitor tablets & $97.24 \pm 1.50$ & $99.30 \pm 1.22$ & 2.42 & 1.51 \\
\hline Lipicure-10 tablets & $99.82 \pm 1.05$ & $99.92 \pm 0.85$ & 0.65 & 1.53 \\
\hline
\end{tabular}

a Values are mean of five determinations \pm SD.

b The tabulated values at $95 \%$ confidence limit are 2.78 and 6.39 , respectively. 
designed the study, participated in the results discussion and prepared the manuscript. All authors read and approved the final manuscript.

\section{Competing interests}

The authors declare that they have no competing interests.

Received: 22 May 2011 Accepted: 7 October 2011

Published: 7 October 2011

\section{References}

1. Kaufman DW, Kelly JP, Rosenberg L, Anderson TE, Mitchell AA: Recent patterns of medication use in the ambulatory adult population of the United States: the Slone survey. JAMA 2002, 287:337-344.

2. Lea AP, McTavish D: Atorvastatin: A review of its pharmacology and therapeutic potential in the management of hyperlipidaemias. Drugs 1997, 53:828-847.

3. The United States Pharmacopoeia 34, NF 29: United States Pharmacopoeia Convention, Mack, Easton, PA; 2011, 1949.

4. Darwish IA, Abdine HH, Amer SM, Al-Rayes LI: Spectrophotometric study for the reaction between fluvoxamine and 1,2-naphthoquinone-4sulphonate: Kinetic, mechanism and use for determination of fluvoxamine in its dosage forms. Spectrochim Acta A Mol Biomol SpectrosC 2009, 72:897-902.

5. Darwish IA, Hussein SA, Mahmoud AM, Hassan Al: Spectrophotometric determination of $\mathrm{H}(2)$-receptor antagonists via their oxidation with cerium(IV). Spectrochim Acta A Mol Biomol Spectrosc 2008, 69:33-40.

6. Ahmed S, Rasul A, Masood Z: Spectrophotometry in Pharmaceutical Analysis Germany: LAP Lambert Academic Publishing; 2011.

7. Gorog S: Ultraviolet-visible spectrophotometry in pharmaceutical analysis Boca Raton, FL: CRC Press; 1995.

8. Darwish IA, Refaat $\mathrm{H}$, Askal HF, Marzouq MA: Generic nonextractive spectrophotometric method for determination of 4-quinolone antibiotics by formation of ion-pair complexes with beta-naphthol. J AOAC Int 2006, 89:334-340.

9. Stanisz B, Rafa W: Development and validation of UV derivative spectrophotometric assay for determination of atorvastatin in Tablets. Chemia Analityczna 2008, 53:417-428.

10. Mishra P, Gupta A, Shah K: Simultaneous estimation of atorvastatin calcium and amlodipine besylate from tablets. Indian J Pharm Sci 2007, 69:831-833.

11. Khan MR, Jain D: Simultaneous spectrophotometric determination of atorvastatin calcium and amlodipine besylate in tablets. Indian J Pharm Sci 2006, 68:546-548

12. Jadhav SD, Bhatia MS, Thamake SL, Pishawikar SA: Spectrophotometric assays for estimation of atorvastatin calcium from tablet dosage forms. Int J PharmTech Res 2010, 2:1948-1953.

13. Ashour S, Bahbouh M, Khateeb M: A novel use of oxidative coupling reactions for determination of some statins (cholesterol-lowering drugs) in pharmaceutical formulations. Spectrochim Acta A Mol Biomol SpectrosC 2011, 78:913-917.

14. Saradhi SV, Rao GD, Srinivasu P, Venkatesh BSS: Visible spectrophotometric assay for the determination of a lipid lowering drug in pharmaceutical dosage forms. J Pure Appl Microbiol 2007, 1:313-315.

15. Nagaraju P, Gopal NV, Srinivas VDN, Padma SVN: Spectrophotometric assays for the determination of atorvastatin calcium in pure and it's pharmaceutical dosage forms. Asian J Res Chem 2008, 1:64-66.

16. Erk N: Extractive spectrophotometric determination of atorvastatin in bulk and pharmaceutical formulations. Anal Lett 2003, 36:2699-2711.

17. Darwish IA: Development and validation of spectrophotometric methods for determination of fluoxetine, sertraline, and paroxetine in pharmaceutical dosage forms. J AOAC Int 2005, 88:38-45.

18. Frag EY, Mohamed GG, Farag AB, Yussof EB: Utility of $\pi$ and $\sigma$-Acceptor Reagents for the Spectrophotometric Determination of Cefotaxime Sodium Antibacterial Drug via Charge Transfer Complex Formation. Insight Pharmaceutical Sciences 2011, 1:47-54.

19. Zayeda MA, Khalilb SM, El-qudabyb HM: Spectrophotometric study of the reaction mechanism between DDQ $\Pi$ - and iodine $\sigma$-acceptors and chloroquine and pyrimethamine drugs and their determination in pure and in dosage forms. Spectrochimica Acta Part A: Molecular and Biomolecular Spectroscopy 2005, 62:461-465.
20. Darwish IA: Kinetic spectrophotometric assays for determination of trimetazidine dihydrochloride. Anal Chim Acta 2005, 551:222-231.

21. Darwish IA: Analytical study for the charge transfer complexes of losartan potassium. Anal Chim Acta 2005, 549:212-220.

22. Darwish $\mid \mathrm{A}$, Refaat $\mathrm{IH}$ : Spectrophotometric analysis of selective serotonin reuptake inhibitors based on formation of charge-transfer complexes with tetracyanoquinodimethane and chloranilic acid. J AOAC Int 2006, 89:326-333.

23. Darwish IA, Abdel-Wadood HA, Abdel-Latif NA: Validated spectrophotometric and fluorimetric assays for analysis of clozapine in tablets and urine. Annali di Chim 2005, 95:345-356.

24. Fidler AT, Baker EL, Letz RE: Neurobehavioural effects of occupational exposure to organic solvents among construction painters. $\mathrm{Br} J$ Ind Med 1987, 44:292-308.

25. Wennborg $H$, Bonde JP, Stenbeck M, Olsen J: Adverse reproduction outcomes among employees working in biomedical research laboratories. Scand J Work Environ Health 2002, 28:5-11.

26. Lindbohm ML, Taskinen H, Sallmén M, Hemminki K: Spontaneous abortions among women exposed to organic solvents. Am J Ind Med 1990, 17:449-463.

27. Wennborg H, Bodin L, Vainio H, Axelsson G: Pregnancy outcome of personnel in Swedish biomedical research laboratories. J Occup Environ Med 2000, 42:438-446.

28. Kristensen P, Hilt B, Svendsen K, Grimsrud TK: Incidence of lymphohaematopoietic cancer at a university laboratory: a cluster investigation. Eur J Epidemiol 2008, 23:11-15.

29. Job P: Ann. Chem. 16 (1936) 97. Advanced Physicochemical Experiments. 2 edition. Oliner and Boyd, Edinburgh; 1964, 54.

30. Askal HF: Spectrophotometric study of the charge transfer complexes of some pharmaceutical butyrophenones. Talanta 1997, 44:1749-1755.

31. Darwish IA, Mahmoud AM, Al-Majed AR: A novel analytical approach for reducing the consumption of organic solvents in the charge transferbased spectrophotometric analysis: application in the analysis of certain antihypertensive drugs. Acta Pharm 2010, 60:493-501.

32. Taha A, Rücker G: Utility of pi-acceptors in alkaloid assay. Arch Pharm (Weinheim) 1977, 310:485-494.

33. Saleh GA, Askal HF, Darwish IA, El-Shorbagi AN: Spectroscopic analytical study for the charge-transfer complexation of certain cephalosporins with chloranilic acid. Anal Sci 2003, 19:281-287.

34. Foster R: Organic charge-transfer complexes. London, New York, Academic Press; 1969, 470

35. The United States Pharmacopeia 24, The National Formulary 19: United States Pharmacopeial Convention, Inc., Rockville; 2008.

36. Nakamura K, Masuda H, Kariyazono H, Arima J, Iguro Y, Yamada K, Sakata R: Effects of atorvastatin and aspirin combined therapy on inflammatory responses in patients undergoing coronary artery bypass grafting. Cytokine 2006, 36:201-210.

37. Athyros VG, Papageorgiou AA, Athyrou W, Demitriadis DS, Kontopoulos AG: Atorvastatin and micronized fenofibrate alone and in combination in type 2 diabetes with combined hyperlipidemia. Diabetes Care 2002, 25:1198-1202.

38. Ballantyne CM, Houri J, Notarbartolo A, Melani L, Lipka LJ, Suresh R, Sun S, LeBeaut AP, Sager PT, Veltri EP, Group ES: Effect of ezetimibe coadministered with atorvastatin in 628 patients with primary hypercholesterolemia: a prospective, randomized, double-blind trial. Circulation 2003, 107:2409-2415.

39. Devabhaktuni M, Bangalore S: Fixed combination of amlodipine and atorvastatin in cardiovascular risk management: patient perspectives. Vasc Health Risk Manag 2009, 5:377-387.

40. Mishra P, Gupta A, Shah K: Simultaneous estimation of atorvastatin calcium and amlodipine besylate from tablets. Indian J Pharm Sci 2007, 69:831-833.

doi:10.1186/1752-153X-5-57

Cite this article as: Wani et al:: Novel microwell-based

spectrophotometric assay for determination of atorvastatin calcium in its pharmaceutical formulations. Chemistry Central Journal 2011 5:57. 